\title{
The Effect of Hurdling Performance on The Adaptive Profile of Cerebral Blood Flow in Vestibular Irritation
}

\author{
${ }^{1}$ Anton Alexandrovich Semchenko ${ }^{(i)}$, ${ }^{2}$ Anna Valerievna Nenasheva ${ }^{(\mathbb{D}}$, ${ }^{3}$ Seyed \\ Morteza Tayebi ${ }^{\circledR},{ }^{1}$ Alexey Viktorovich Savchenkov \\ ${ }^{1}$ Vocational Pedagogical Institute, South Ural State Humanitarian Pedagogical University, Chelyabinsk, \\ Russia. ${ }^{2}$ Theory and Methods of Physical Education and Sport Department, the Institute of Sports, Tourism \\ and Service, South Ural State University (National Research University), Chelyabinsk, Russia. ${ }^{3}$ Department \\ of Exercise Physiology, Faculty of Sport Science, Allameh Tabataba'i University, Tehran, Iran.
}

Submitted 13 July 2021; Accepted in final form 20 October 2021.

\begin{abstract}
Background. The reactions of the whole organism that occur under the action of accelerations of rectilinear and rotational motion are factors that have a systemic effect on the cerebral blood flow. The use of hurdling performance in the structure of human physical activity can have a significant impact on the tone of the brain vessels, their elasticity and venous outflow in the development of fatigue caused by statokinetic stimuli. Objectives. The research purpose is to investigate the adaptive profile of cerebral blood flow in the implementation of statokinetic reactions in individuals engaged in hurdling performance. Methods. 108 university students took part in the study on a voluntary basis. All students were randomly divided into two groups. The adaptive profile of the cerebral blood flow was evaluated using rheoencephalography under the conditions of stabilometric testing at the beginning of the school year, after three and six months. Results. It is shown that a more perfect adaptation of the cerebral blood flow to vestibular irritation when barrier running exercises are included in the structure of physical activity is due to adequate stimulation of ampullary receptors caused by head tilts and contralateral synchronization of the muscles of the upper and lower extremities, which determine the kinematic structure of movements to overcome the barrier bar in conditions of rectilinear and nonlinear accelerations. Conclusion. Our study revealed the process of developing stable compensatory reactions of cerebral blood flow in students during systematic hurdling performance.
\end{abstract}

KEYWORDS: Hurdling Performance, Postural Stability, Stabilometry, Cerebral Hemodynamics.

\section{INTRODUCTION}

The vessels of the brain and the receptors of the vestibular apparatus in the implementation of statokinetic reactions are in a state of physiological arousal with the corresponding activation of the mechanisms of metabolism. The increasing lack of oxygen and energy supply of the brain against the background of vestibular irritation leads to a violation of the cerebral blood flow, which leads to an increase in the level of under-oxidized products in the tissues, the accumulation of which is a threat to the homeostasis of the body as a whole.
Modern studies have found that physical exercise activates changes in the properties of brain vessels (1). It is shown that these changes in physical activity of different directions are specific, and therefore this issue remains debatable (2). Data on adaptive heart responses in the implementation of statokinetic responses in athletes and individuals with somatosensory disorders are presented in detail (3). However, the task of finding effective means of physical education for the development of long-term adaptation of

*. Corresponding Author:

Anton Alexandrovich Semchenko, Ph.D.

E-mail: semchenkoaa@bk.ru 
the blood supply system of the brain in vestibular stimuli remains urgent.

Hurdling as a physical exercise is characterized by a cyclically repeated complex coordination structure of supporting and unsupported body positions (4). The nature of the impact of such a load on the human body is determined by the combined effect of muscular and vestibular loads, which leads to the study of potential hemodynamic adaptive reactions to stimuli that occur during active and passive movements in space in people engaged in barrier running.

It seems that the use of barrier running in the structure of human physical activity has a positive effect on the tone of the brain vessels, their elasticity and venous outflow in the development of fatigue caused by vestibular irritations. So, the aim of the study is to investigate the adaptive profile of cerebral blood flow in the implementation of statokinetic reactions in individuals engaged in hurdling.

\section{MATERIALS AND METHODS}

Ethical Considerations. The study was conducted in accordance with the Council of Europe Convention on Human Rights and Biomedicine (1999) and the Helsinki Declaration of the World Medical Association (2013 edition). The study included healthy volunteers in accordance with the approval granted by the Ethical Committee of the University, prior to the study the participants' informed consent was obtained.

Participants. The instrumental study was conducted at the Sports Science Research Center of the South Ural State University. The study involved 108 apparently healthy students of the South Ural State Humanitarian and Pedagogical University aged 18-20 years. All students were randomly divided into two groups (SG-I and SGII). SG-I $(n=54)$ - students engaged in the standard physical education program for universities; SG-II $(n=54)$ - students engaged in a physical education program based on the use of barrier running exercises. At all stages of the study, the participants were acquainted with study procedures.

Protocols. In order to simulate statokinetic reactions, the sensory-vestibular method of stabilometry was used with the "MBN Stabilo" platform and a 30-second rheoencephalogram (REG) recording in the following positions: the main two-legged position with open eyes; head turn to the left; head turn to the right; main two- legged position with closed eyes; head turn to the left with closed eyes; head turn to the right with closed eyes.

Before and after vestibular stimulation 4 hemodynamic parameters of cerebral blood flow (rheographic index (RI); modulus of elasticity; dicrotic index (DI); diastolic index (DaI)) were obtained.

The scheme of applying the rheograph electrodes to the fronto-mastoid (FM) lead, which characterizes the blood flow in the carotid basin of the brain, was used.

The resulting data array was processed and presented in accordance with the generally accepted standards of the International Society for Posture and Gait Research.

The visual characteristics of the examined sample excluded pathological diseases. The group of examined hurdlers was characterized by left-sided motor asymmetry of the legs and rightsided motor asymmetry of the arms.

Statistical Analysis. The data obtained in our study were processed with the $\mathrm{R}$ software package (version 3.5.0). The type of feature distributions was calculated using the ShapiroWilk W-test. The differences were considered significant when the level of statistical significance was less than 0.05 .

\section{RESULTS}

Table 1 shows the results of rheoencephalographic investigation in students of the studied groups when modeling vestibular irritation in conditions of short-term adaptation to the loads of running with barriers.

Figure 1 reflects the change in the parameters of the adaptive profile of cerebral blood flow in students aged 18-20 years when modeling vestibular irritation in conditions of long-term adaptation to the loads of running with barriers.

Figure 2 In Figure 2, changes in the statokinesiogram and stabilogram of 18-20-yearold students are shown when adapting to the physical activity associated with hurdling.

\section{DISCUSSION}

The ability of a person to tolerate vestibular stimuli (accelerations, decelerations, shocks, etc.) is considered by researchers as a necessary condition for adaptation to the action of social, labor, and extreme factors $(5,6)$. The central nervous system plays a significant role in the genesis of the body's responses to vestibular irritation (7). Modeling of vestibular stimuli is 
one of the ways to study the work of the brain in various aspects, including: in the study of vascular reactions and blood supply mechanisms (8).

Table 1. Dynamics of cerebral blood flow indicators in 18-20-year-old students under conditions of vestibular irritation after 12 weeks of barrier running

\begin{tabular}{ccc}
\hline & Background Values & Vestibular Irritation - 3' \\
\hline SG-I (n= 54) & & \\
RI, Ohm & $0,12 \pm 0,01$ & $0,13 \pm 0,011^{*}$ \\
MU, \% & $16,3 \pm 0,3$ & $17,6 \pm 0,5^{*}$ \\
DI, \% & $55,7 \pm 1,1$ & $61,5 \pm 1,3^{*}$ \\
DaI, \% & $75,4 \pm 2,3$ & $77,3 \pm 2,5$ \\
SG-II (n= 54) & & \\
RI, Ohm & $0,13 \pm 0,012$ & $0,15 \pm 0,016^{*}$ \\
MU, \% & $16,4 \pm 0,1$ & $16,8 \pm 0,1$ \\
DI, \% & $55,3 \pm 1,3$ & $58,3 \pm 0,9$ \\
DaI, \% & $75,2 \pm 2,1$ & $75,7 \pm 1,8$ \\
\hline
\end{tabular}

RI: Rheographic Index; MU: Modulus of Elasticity; DI: Dicrotic Index; DaI: Diastolic Index; **: statistically different; p<0.01; *: statistically different, $\mathbf{p}<\mathbf{0 . 0 5}$.
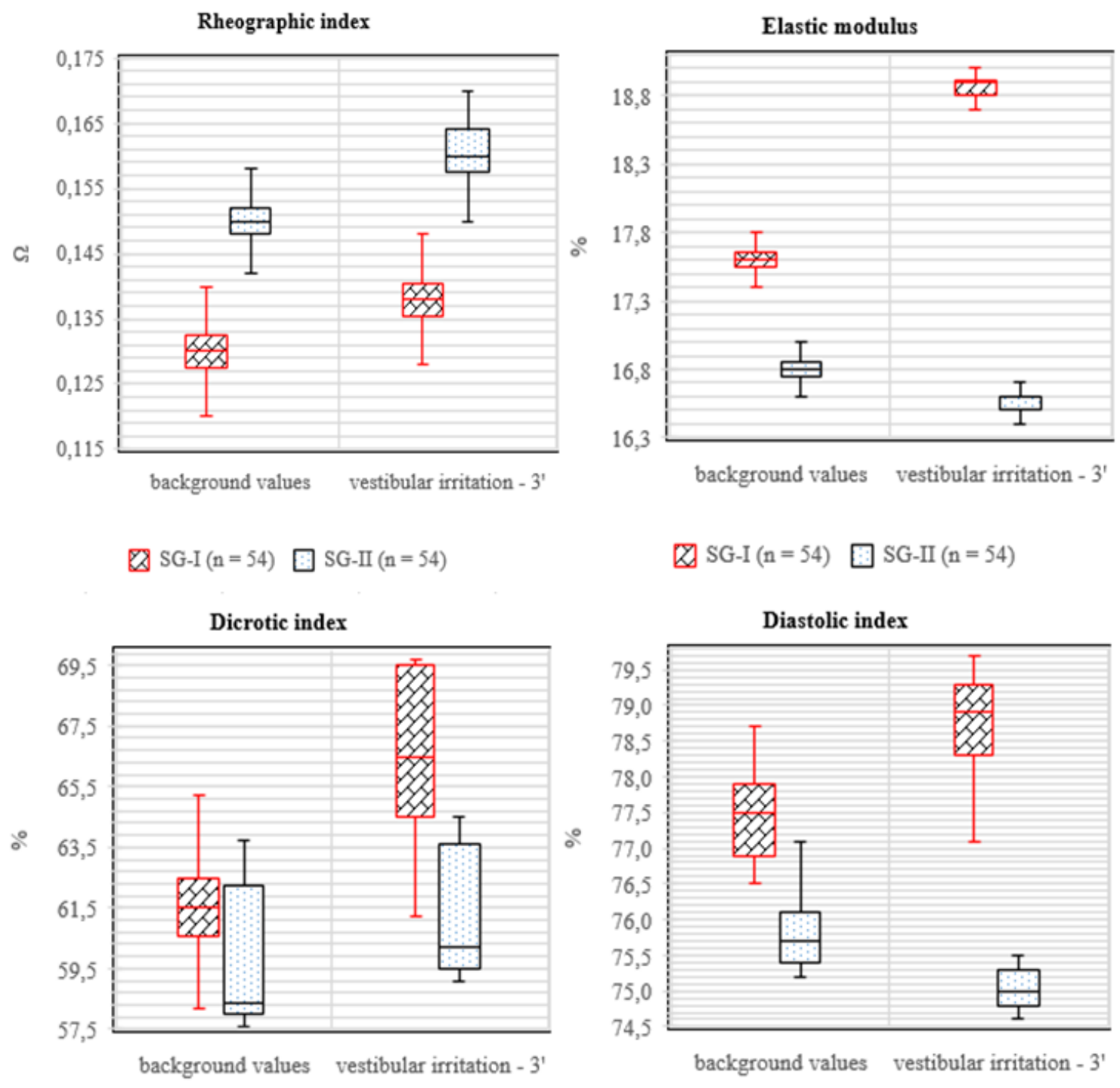

Figure 1. The profile of cerebral blood flow in students aged 18-20 years after 24 weeks of hurdling. 


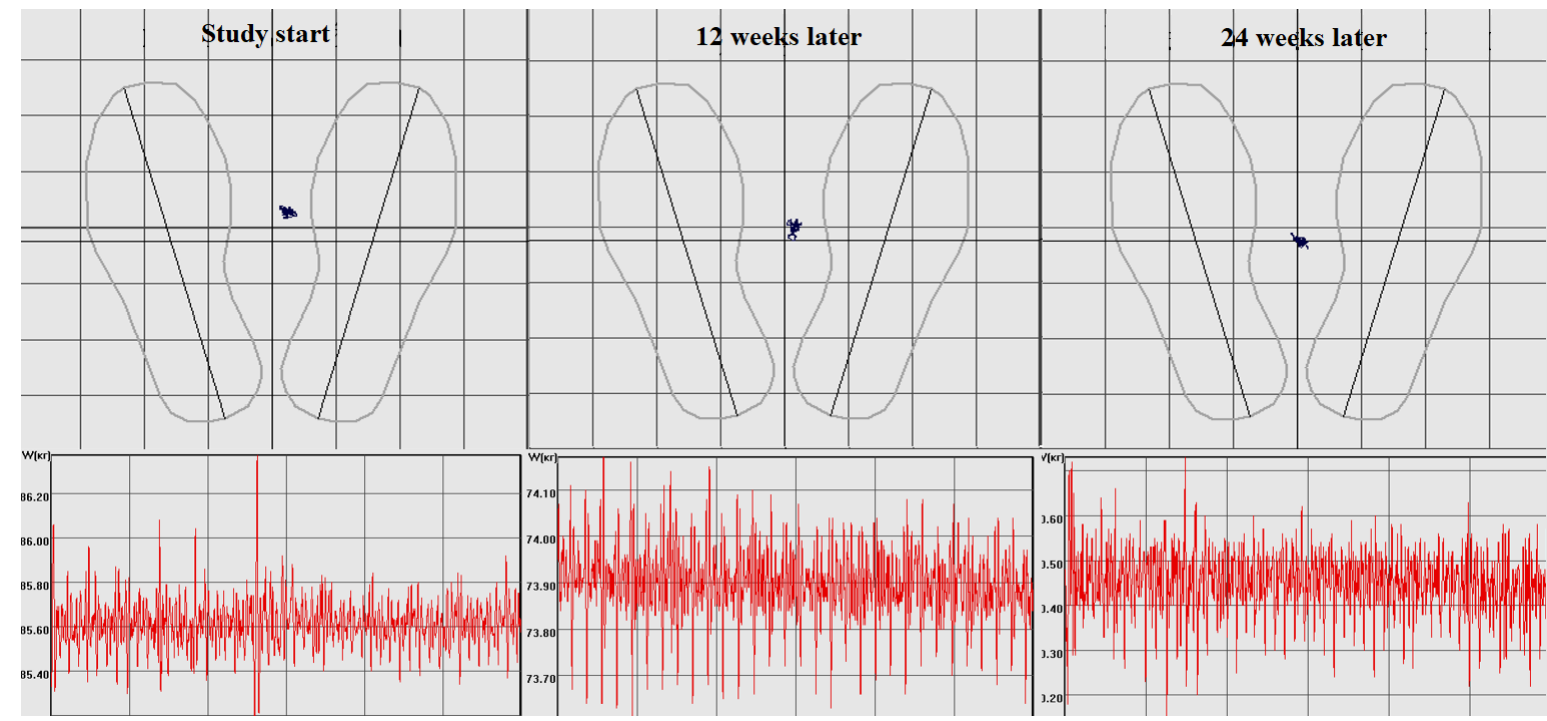

Figure 2. Averaged models of statokinesiograms and stabilograms in students in the dynamics of adaptation to loads in hurdling.

It is known that the hemodynamics of the brain in comparison with other internal organs is characterized by relative constancy and is practically not affected by various physiological factors, including those associated with the performance of physical exercises (9). However, more recent studies suggest that somatosensory cortical neuronal activity and increased brain metabolism as associated effects of exercise may lead to functional changes in cerebral blood flow (10). The inconsistency of the data in this issue actualizes the task of physiological justification of effective means of physical education for optimizing the activity of the central nervous system organs in the implementation of statokinetic reactions and increasing the stability of the cerebral blood flow to all kinds of statodynamic influences.

It is shown that the rheoencephalographic parameters of the adaptive profile of the cerebral blood flow in mature people without chronic diseases are characterized by a steep rise of the anacrota, a pronounced amplitude, a gentle descent and one distinct dicrotic wave (11).

Our study showed that the average group indicators of the rheographic index in students aged 18-20 at the beginning of the academic year were within the lower limit of the physiological norm (Table 1). After 12 and 24 weeks of systematic physical activity, including barrier running and its elements, the rheographic index for vestibular irritation increased $(p<0.01)$. The tendency to increase the rheographic index within the reference values indicates a change in the blood filling of the vessels of the carotid basin (12).

Similar, but less pronounced dynamics of the rheographic index indicators during statokinetic stimulation was also observed in students whose physical activity structure did not include the load associated with hurdling ( $<$ 0.05) (Table 1). In the course of the study, it was found that when using standard physical education programs for students, according to the indicators of pulse blood filling, there is a more pronounced hypovolemia of the blood vessels of the carotid basin of the brain compared to students who have loads in hurdling (Figure 1).

Thus, it can be assumed that the formation of a systemic structural trace during adaptation to the loads of running with barriers is characterized by a gradual optimization of pulse blood filling in the vessels of the carotid basin of the brain.

Vestibular irritation in the group of 18-20year-olds who did not have a load in hurdling was accompanied by a decrease in the elasticity of the vessels of the carotid basin of the brain ( $p<0.05)$, as evidenced by a decrease in the group's average modulus of elasticity (Table 1).

The values of the coefficient of elasticity of the vascular wall in students in the SG-I sample after 24 weeks from the beginning of the academic year exceeded the standard indicators by $4.4 \%$. At the same time, after 6 months of physical exercises with elements of barrier running, the coefficient of elasticity of the vascular wall in the SG-II 
sample increased compared to the background values at the beginning of the study and did not change under the influence of vestibular stimuli (Figure 1).

It can be noted that a sample of students aged 18-20 years is characterized by an increase in the tone of the cerebral vessels during the academic year, which indicates an increasing level of neuropsychiatric stress. The hypovolemia of the brain vessels detected during this period according to rheoencephalography can act as a compensatory reaction in response to neuropsychiatric stress caused by factors that accompany the educational process at the university (increased influence of mental stress, lack of motor activity, exam stress, sleep and nutrition disorders, etc.) $(13,14)$.

The value of the average group indicator of the dicrotic index in the SG-I sample was lower than the values of the control group $(p<0.05)$ when modeling functional samples on the stabiloplatform at the final stage of the study. Prolonged (24 weeks) use of physical activity associated with barrier running has an effect on reducing the tone of the vascular walls of the microcirculatory bed of the carotid basin of the brain, as evidenced by the dynamics of the dicrotic index in the SG-II sample (Figure 1).

It was found that the value of the dicrotic index in students aged 18-20 years, engaged in standard physical education programs, by the end of the first half of the year exceeded the reference values by $5.1 \%$. The researchers noted that the solution of cognitive tasks correlates with changes in the course of individual nervous processes and disorders of the blood supply system. The method of rheoencephalography allows us to identify these changes and to take timely corrective measures to ensure intellectual activity and the preservation of the level of health of the student population.

Vestibular irritation did not significantly affect the hemodynamic responses associated with the provision of venous outflow in the brain in the students in the SG-II sample (Table 1).

In contrast, the SG-I sample showed an increase in the diastolic index $(\mathrm{p}<0.05)$ (Figure 1). The latter circumstance indicates a slight obstruction of venous outflow in the vascular pool of the carotid arteries of the brain in students aged 18-20 years with vestibular irritations, which indicates an insufficient level of statokinetic stability. Obstruction of venous outflow may be a consequence of blood deposition in the extremities and insufficient blood supply to the brain (15), which leads, as a result, to insufficient statokinetic stability.

Stabilometric indicators of 18-20-year-old students in the SG-II sample with vestibular irritation had the same tendency to escalate the proportion of medium (from 0.5 to $1.5 \mathrm{~Hz}$ ) frequency fluctuations of the statokinesiogram during unstable adaptation to loads in hurdling and an increase in the proportion of slow high amplitude (from 0 to $0.3 \mathrm{~Hz}$ ) frequency fluctuations-with the development of long-term adaptation ( $\mathrm{p}<0.05$ ) (Figure 2), which indicates an increase in statokinetic stability under the influence of barrier running.

An increase in statokinetic stability is a necessary condition for stabilizing a high level of mental performance and productivity in performing cognitive tasks (16). High resistance to vestibular irritation is one of the factors of increasing the plasticity of the brain (17), which is especially important at the stage of education.

Our study shows that vestibular stimuli when including systematic barrier running and its elements in the structure of the physical education program at the university do not have a significant effect on the deterioration of hemodynamic reactions and contribute to the improvement of cerebral blood flow in terms of rheoencephalography in the FM-lead.

\section{CONCLUSION}

A better adaptation of the cerebral blood flow to vestibular stimulation when barrier running exercises are included in the structure of physical activity may be due to adequate stimulation of ampullary receptors caused by head tilts and contralateral synchronization of the muscles of the upper and lower extremities, which determine the kinematic structure of movements to overcome the barrier bar in conditions of rectilinear (30-110 meters of hurdling) and nonlinear accelerations (> 200 meters of hurdling).

The change in a number of rheoencephalography indicators in students compared to the reference values indicates a tense state of the cerebral blood flow in the dynamics of the academic year. Barrier running classes and the inclusion of its elements in the structure of physical activity cause students to increase the excitability of the central nervous system. Vestibular irritation, which occurs in students with continuous work of a static and dynamic 
nature, causes the development of mental fatigue. Mental fatigue impairs the ability to subjectively perceive one's mistakes and generally disrupts the functioning of stimulus response systems.

Our study revealed the process of developing stable compensatory reactions of cerebral blood flow in students aged 18-20 years during systematic barrier running classes aimed at maintaining statokinetic stability and mental performance in the conditions of vestibular irritation.

\section{APPLICABLE REMARKS}

- This study implies that the use of specific exercises in physical education of university student can improve intermuscular coordination, statokinetic balance, body balance and cerebral hemodynamics. Thus, mental performance and resistance to exam stress are increased.

\section{CONFLICT OF INTERESTS}

The authors claim that there is no known conflict of interests.

\section{ACKNOWLEDGMENTS}

This work was supported by the Shadrinsk State Pedagogical University of the Ministry of Education of the Russian Federation [Grant No. ShK-027-22].

The work was supported by Act 211 Government of the Russian Federation, contract № 02.A03.21.0011.

\section{REFRENCES}

1. Hiura M, Nariai T, Ishii K, Sakata M, Oda K, Toyohara J, et al. Changes in cerebral blood flow during steady-state cycling exercise: a study using oxygen-15-labeled water with PET. J Cereb Blood Flow Metab. 2014;34(3):389-96. doi:10.1038/jcbfm.2013.220 pmid: 24301294

2. Ivanovna PV, Andreevich SD, Germanovna KY, Anatolyevna BN, Vitalyevich LJ, et al. Psychophysiological Determinants of Successful Training and Competitive Activity of Martial Artists. Journal of Pharmaceutical Sciences and Research; Cuddalore. 2017;9(10):1792-6.

3. Cherepov E, Epishev V, Terekhina E. Effects of modern fitness technologies on physical qualities in students with locomotor disorders. Minerva Ortop Traumatol 2018;69(Suppl. 1 al N. 3):43-8. doi:10.23736/S0394-3410.18.03879-1

4. Semchenko AA, Nenasheva AV. Influence of Long-Term Motor Specialization in Barrier Running on Biokinematic Indicators of Normal Statics of the Musculoskeletal System. Human Sport Medicine. 2017; S:66-72 [Article in Russia]. doi:10.14529/hsm17s07

5. Sishko DV. Description of electric processes of heart at boxers in the conditions of vestibular irritations. Pedagogy, psychology and medico-biological problems of physical education and sports. 2009; 11:1147.

6. Cohen HS. Use of the Vestibular Disorders Activities of Daily Living Scale to describe functional limitations in patients with vestibular disorders. Journal of vestibular research: equilibrium \& orientation. 2014;24(1):33-8. doi:10.3233/VES-130475 pmid: 24594498

7. Chinkin AS, Husnullina RI. Vegetative reactions to vestibular irritation at ski jumping practice. European Journal of Natural History. 2007;4:156-7.

8. Kapilevich LV, Yezhova GS, Zakharova AN, Kabachkova AV, Krivoshchekov SG. Brain Bioelectrical Activity and Cerebral Hemodynamics in Athletes under Combined Cognitive and Physical Loading. Human Physiology. 2019;45(2):164-73. doi:10.1134/S0362119719010080

9. Lassen NA. Cerebral blood flow and oxygen consumption in man. Physiological reviews. 1959;39(2):183-238. doi:10.1152/physrev.1959.39.2.183 pmid: 13645234

10. Cirrik S, Hacioglu G. Neurophysiological Effects of Exercise. In: Sözen H, editor. Fitness Medicine. online: intechopen.com; 2016. p. 2189-281. doi:10.5772/64801 pmid: 27803779

11. Belousova NA, Dolgova VI, Mamylina NV. The influence of physical activity on the condition of the cerebral circulation in female students. International Journal of Pharmacy and Technology. 2016; 4:25303-10.

12. Bodo M, Pearce F, Garcia A, Van Albert S, Settle T, Szebeni J, et al., editors. In vivo cerebral blood flow autoregulation studies using rheoencephalography2010 April 01, 2010: Journal of Physics; Conference Series. doi:10.1088/1742-6596/224/1/012088

13. Kovaleva ME. Special Aspects of Neuropsychic Maladaptation in Specialists of Various Professions. Psychology and Law. 2019;9(3):219-31 [Article in Russia]. doi:10.17759/psylaw.2019090316 
14. Gnatyshina EA, Uvarina NV, Salamatov AA, Savchenkov AV, Pakhtusova NA. Analysis of gender differences in the professional identity indicators of pedagogical university students. Espacios. 2018;39:7-18.

15. Pakula H, Szapiro J. Anatomical Studies of the Collateral Blood Supply to the Brain and Upper Extremity. Journal of Neurosurgery. 1970;32(2):171-9. doi:10.3171/jns.1970.32.2.0171 pmid:5411994 16. Glaznikov LA, Sorokina LA, Buynov LG, Plakhov NN. Constitutional Traits of the Statokinetic Stability of Flying Personnel under the Conditions of Long Navy Trip. Human Physiology. 2019;45(7):793-5. doi:10.1134/S0362119719070053

17. Han BI, Song HS, Kim JS. Vestibular rehabilitation therapy: review of indications, mechanisms, and key exercises. J Clin Neurol. 2011;7(4):184-96. doi:10.3988/jen.2011.7.4.184 pmid: 22259614 